\title{
Improved Energy Management Performance for Hierarchical Routing in WSNs
}

\author{
https://doi.org/10.3991/ijes.v6.i2.8818 \\ Yassine Rayri ( $₫$ ), Hatim Kharraz Aroussi, Abdelaziz Mouloudi \\ Ibn Tofail University, Kenitra, Morocco \\ rayri.yassine@gmail.com
}

\begin{abstract}
The rapidly evolving of technology in the area of communication and information, allowed the creation of a new device called wireless sensor. This small device allows measuring physical phenomenon quantity in environment such as light, pressure, temperature and transforming it autonomously into a digital value, In order to send to one or several points of collections, which is going to be processed. However, a wireless sensor network (WSN) is a network formed by a large number of sensor nodes, which are able to acquire, aggregate compress and to transmit data towards a base station. However, several constraints complicate the deployment and management of wireless sensor networks. The economy of energy is among the major issues of these networks, and it is difficult even impossible to replace the sensors or their battery because of the location of deployment that is often inaccessible. Among Scientifics researches, we find the improvement the energy management in WSNs. In this paper, we propose a new routing protocol based on clustering. The purpose is to have an equal distribution of the energy throughout the network.
\end{abstract}

Keywords-Wireless Sensor, Clustering, Conservation, Energy, Equitable distribution

\section{Introduction}

Wireless sensor is one of many emerging creations of the new technology consisting of spatially distributed autonomous micro devices. This device allows detecting and measuring a physical quantity from the environment such as light, heat, pressure, and transforming it into a digital value that can be processing and routing toward base station. The deployment of several wireless sensors communicating by wireless radio, form a Wireless Sensor Network. WSN considered a special type of ad hoc networks where the fixed communication infrastructure and centralized administration are absent and the nodes assume both the role of hosts and routers. Several constraints prevent proper deployment these networks. One of the most significant challenges for Wireless Sensor Networks is energy consumption. However the economy of energy is among the major issues of these networks, and it is difficult even impossible to replace the sensors or their battery because of the location of deployment that is often inaccessible $[1,2,3]$.The equitable distribution of energy throughout the network is 
another problem, especially when the station base is not in the middle of the network $[10,11]$.In this work, we propose an improvement of routing protocols (called EDE: Equitable Distribution Energy) based on Leach protocol by reducing the overload of Cluster-Head, in order to well distribute the energy overall network, avoid the black holes (death node). This optimization consists to create a new node (Node-Transfer) within the cluster that is only responsible of transferring data into the base station (BS). Our network will be Structure as a producer-consumer pattern. In the end, we show the performance of our proposed protocol by comparing it with other wellchosen protocols (LEACH and SEP).We choose to compare with LEACH because our proposed protocol is an improvement of LEACH protocol, and also, most of the analytical results for LEACH-type schemes are obtained assuming that the nodes of the sensor network are equipped with the same amount of energy (case of homogeneous). And we choose to compare with SEP because it is based on heterogonous sensor networks [9].

\section{$2 \quad$ Hierarchical Routing}

The hierarchical routing allows to partition network into sub-set (cluster) and it based on the concept of member nodes and Cluster-Head $(\mathrm{CH})$ nodes. The member nodes route their messages to the Custer-Head, which then routes this messages in the entire network to the base station (Sink) [4,11]. The main objective of the hierarchical routing is to maintain effectively the energy consumption of sensor nodes in involving those into the communication multi-hop within a cluster and performing the aggregation and fusion of data in order to reduce the number of messages transmitted to the destination $[4,5,11]$.

\subsection{Leach Protocol}

LEACH is one of the hierarchical routing protocols more popular in wireless sensor networks. LEACH protocol was introduced by Heinzelman in 2002. The principle is to form clusters of sensor nodes and then use cluster-Heads premises as gateway to reach the base station. This routing protocol allows conserving energy, because the transmissions are performed only by the cluster-Head rather than by all the sensor nodes $[4,5]$. The protocol takes rounds; each round consists of two phases [6,7]:

- Set-up

- Steady-state

\subsection{SEP Protocol}

SEP, for electing cluster heads in a distributed fashion in two-level hierarchical wireless sensor networks [9]. Unlike prior work, SEP is heterogeneous-aware, in the sense that election probabilities are weighted by the initial energy of a node relative to that of other nodes in the network. This prolongs the time interval before the death of 
the first node, which is crucial for many applications where the feedback from the sensor network must be reliable. This protocol improves the stable region of the clustering hierarchy process using the characteristic parameters of heterogeneity, namely the fraction of advanced nodes $(\mathrm{m})$ and the additional energy factor between advanced and normal nodes $(\alpha),[9][12]$.

\section{Proposed Work}

\subsection{Problematic}

Problem 1: (Why overload the Cluster-Head?): Generally, the Cluster-Head is the most active node of the cluster, because it manages all nodes member in the cluster. In effect, after the election of the Cluster-Head, it is in charge of the creation and the organization of the cluster, and then the data collection, the treatment and the compression of these data in order to transmit them to the Base Station [10,11]. In carrying out all these stains, the Cluster-Head loses a lot of energy, so that result a quick death of the cluster head. Therefore, this leads to have holes (dead nodes) in the whole of the network [10].

Problem 2: (The location of the BS): The sensors elute Cluster-Head who are located far from the BS consume more energy, because the transmission distance to the BS is important. As well, the areas that consist of these sensors die entirely. Therefore, when we work in the large area and especially when we have an important number of nodes, the main sensors in the network (far from BS) loses a lot of energy $[8,10]$.In this case, we can say that we do not have an equitable energy distribution over all the nodes of the network.

\subsection{Proposed Solution}

To solve these problems, we propose to create a new node that only takes care of the transfer of data to the base station. We manage the communication mechanism between the different network parts with the producer-consumer pattern.

Producer-Consumer pattern: The producer-consumer model consists of sharing the same buffer for two processes.

- The producer produces objects that he deposits in the buffer.

- The consumer removes objects from the buffer to consume them.

Problems:

- The producer wants to deposit an object while the buffer is already full.

- The consumer wants to remove an object from the buffer while the one is empty.

- The producer and the consumer must not simultaneously access the buffer [14].

EDE Protocol (Node-Transfer): To reduce the functionalities of the ClusterHead, it has established a node that is only responsible of the transfer of the data. The 
cluster-head delegates the task of the transmission of packets to the base station to a node of the cluster. [11]

In this case, a cluster schematize as a producer-consumer pattern as shown in Fig.1. The member nodes are the producers, the Cluster-Head is the buffer whose data processes, aggregate and compress, and the consumer is the transfer node. The latter, that will pick up the data from the Cluster - Head to transfer them to the BS.

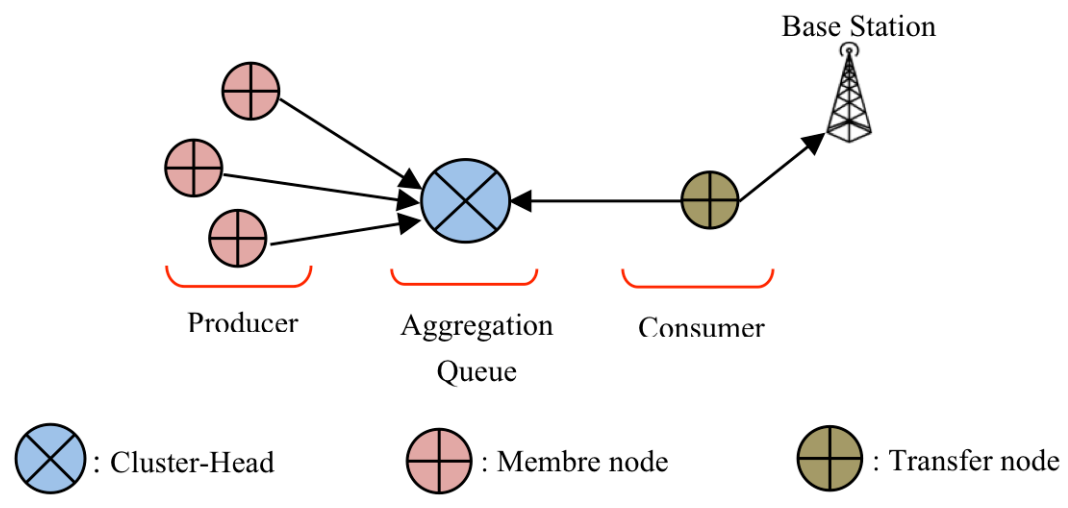

Fig. 1. Producer-consumer pattern apply to cluster

In a defined cluster Fig.2, the energy capacity of the chosen Node Transfer must be greater than the energy of the $\mathrm{CH}$. If there is not a node that has energy higher than the $\mathrm{CH}$, we look for a node that has the same energy as the $\mathrm{CH}$. Otherwise; the latter will be node-transfer. If we have a several nodes with the same energy in the cluster, we choose one of them randomly.

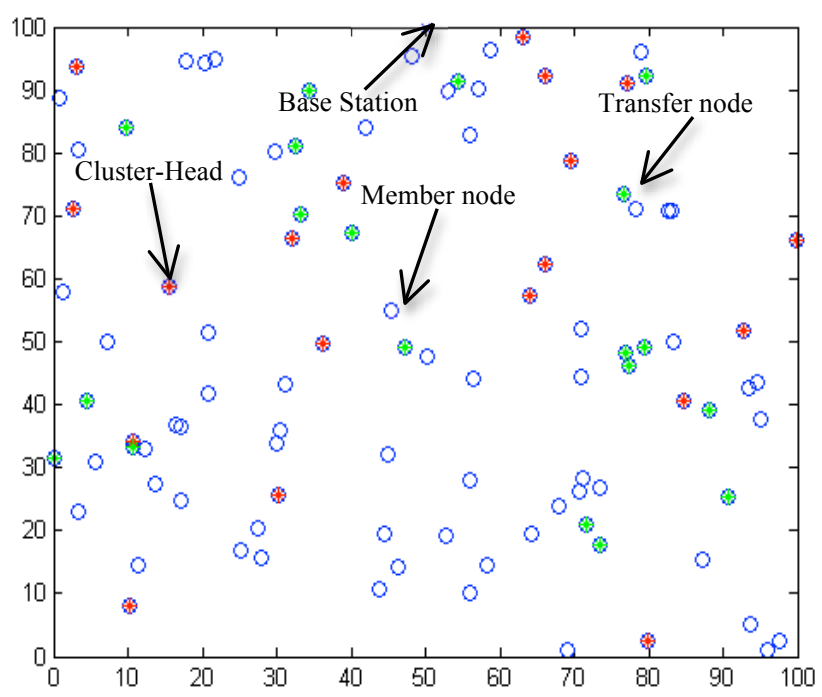

Fig. 2. Random distribution of 100 sensor nodes with EDE 


\section{Simulation \& Results}

The wireless sensors deployed on an area of $100 \mathrm{~m} * 100 \mathrm{~m}$ with a base of station located at the point $(50,100)$.

Table 1. Parameters table

\begin{tabular}{|l|l|}
\hline Initial energy of nodes & $0.25 \mathrm{joul}$ \\
\hline Transmitter and receiver energy & $50 \mathrm{nj} / \mathrm{bit}$ \\
\hline Aggregation energy & $5 \mathrm{nj} / \mathrm{bit}$ \\
\hline Data packet length & $4000 \mathrm{bit}$ \\
\hline $\mathbf{E}_{\mathrm{fs}}$ & $10 \mathrm{pj} / \mathrm{bit} / \mathrm{m} 2$ \\
\hline $\mathbf{E}_{\text {amp }}$ & $0.0003 \mathrm{pj} / \mathrm{bit} / \mathrm{m} 4$ \\
\hline
\end{tabular}

\subsection{Compare with Leach Protocol}

In this simulation, we compare our protocol EDE with Leach protocol the distribution (position) of dead nodes in the surface of the network. We consider that all nodes have an equal initial energies and each death node excluded from the next round.

In Fig.3, where treated by the classical Leach protocol, shows clearly, that the lower part of the network represents a black area of which all sensors are dead. Thus, we may not have the information of this area that is not covered.

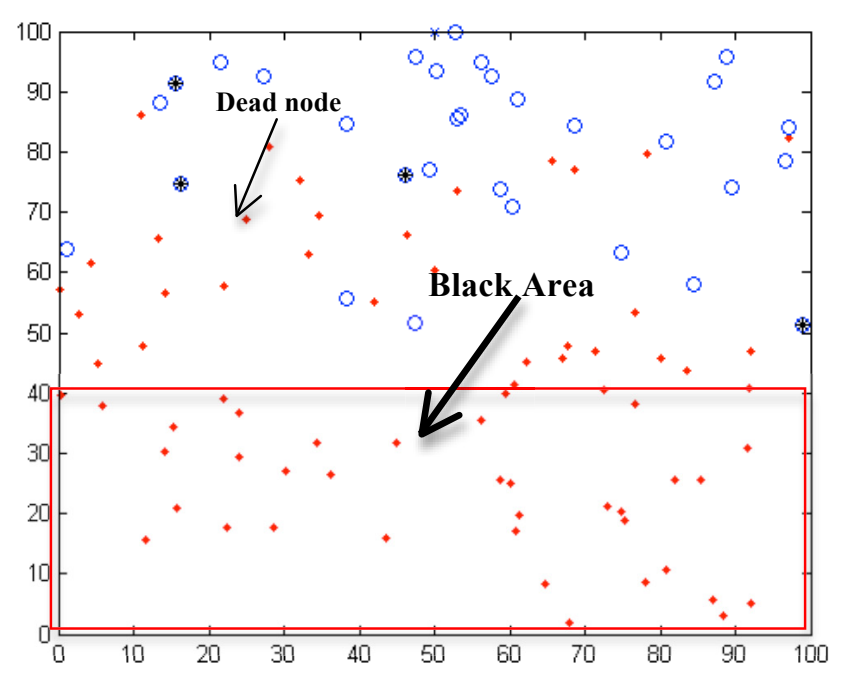

Fig. 3. Simulation with LEACH

Fig.4 illustrates, treated by EDE in which we show the death of the nodes well distributed across the network. Consequently, we can have a data collection on the whole area of the network. 
Short Paper-Improved Energy Management Performance For Hierarchical Routing in WSNs

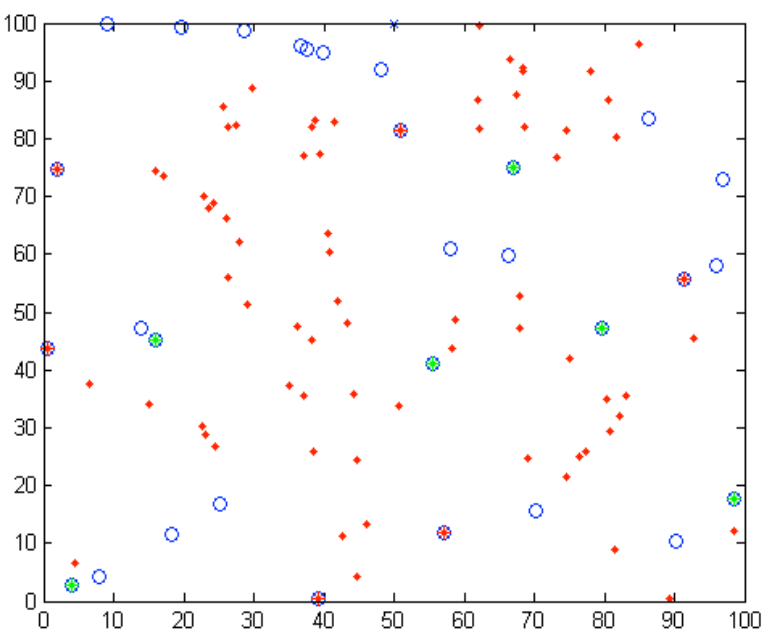

Fig. 4. Simulation with EDE

\subsection{Compare with SEP Protocol}

Here in Fig.5, we compare our EDE protocol with the SEP protocol with $\alpha=0.2$ and $\mathrm{m}=1$ :

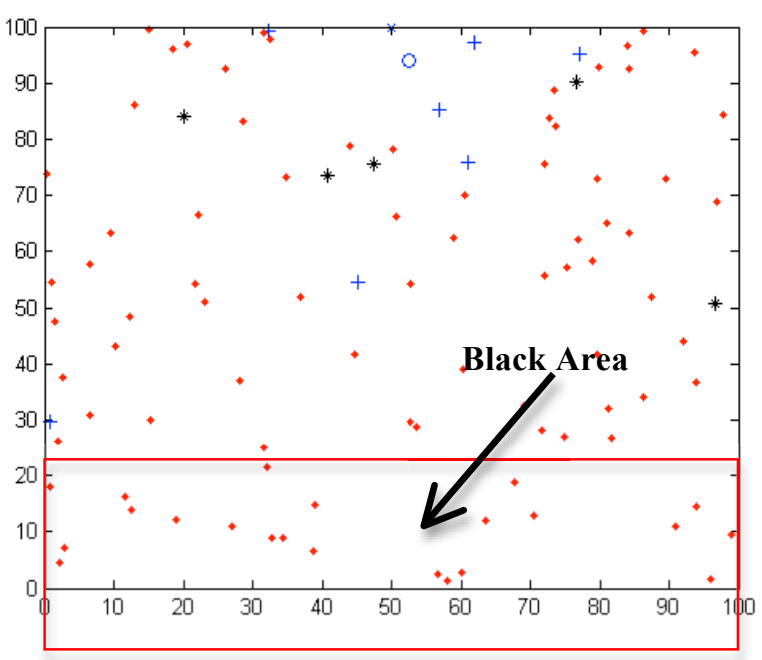

Fig. 5. Simulation with SEP 


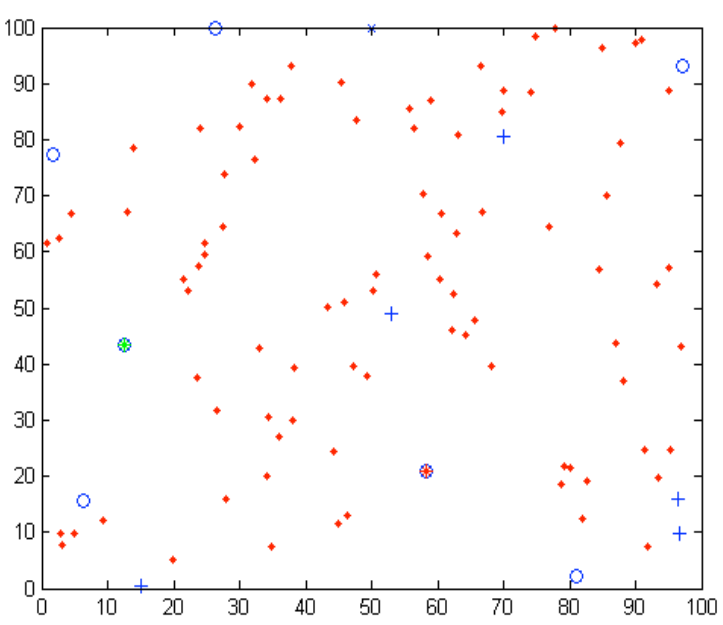

Fig. 6. Simulation with EDE (Advanced)

Note also, for SEP, the energy distribution is fair across the network, contrary to our proposal. Moreover for the SEP protocol, the normal nodes died first, while for the proposed protocol, as shown in Fig.6, it remains the two categories of nodes, normal and advanced. This implies that we do not have an overload on some nodes.

\section{Conclusion \& Perspective}

The Wireless Sensor Networks have been the object of several studies and research to solve the problems that prevent and stabilize the good design of these networks. Often, the scientific researches in the area of energy conservation focus on maximizing the lifetime of wireless sensors. However, we must not forget the good distribution of energy on the whole of the network.

The integration of the transfer node (Node-Transfer) and producer-consumer pattern in this proposed work EDE (Equitable Distribution Energy) have exposed good fair distribution energy on the whole of the network.

Although, the results of energy distribution are good, we are now improving our EDE protocol to have better results in energy conservation.

\section{References}

[1] Loren P. Clarea, Gregory J. Pottieb and Jonathan R. Agrea. "Self-Organizing Distributed Sensor Networks" Proc. SPIE 3713, Unattended Ground Sensor Technologies and Applications, (July 30, 1999), 229.

[2] Chunyao FU, Zhifang JIANG, Wei WEI, Ang WEI "An Energy Balanced Algorithm of LEACH Protocol in WSN", IJCSI International Journal of Computer Science Issues, Vol. 10, Issue 1, No 1, January 2013. 
[3] J.Gnanambigai, Dr. N.Rengarajan, K. Anbukkarasi. "Leach and Its Descendant Protocols: A Survey". International Journal of Communication and Computer Technologies Volume 01 - No.3, Issue: 02 September 2012.

[4] Khushboo Pawar, Y. Kelkar. "A Survey of Hierarchical Routing Protocols in Wireless Sensor Network". International Journal of Engineering and Innovative Technology (IJEIT) Volume 1, Issue 5, May 2012.

[5] Xu-XunLiu. "A Survey on Clustering Routing Protocols in Wireless Sensor Networks". 9 August 2012, Sensors 2012, 12, 11113-11153. https://doi.org/10.3390/s120811113

[6] W.R. Heinzelman, A. Chandrakasan, and H. Balakrishnan, "Energy-efficient Communication Protocols for Wireless Microsensor Networks", in Proc.33rd Hawaii International Conference on System Sciences, vol. 2, January 2000. https://doi.org/10.1109/ HICSS.2000.926982

[7] W.B. Heinzelman, A. Chandrakasan, and H. Balakrishanan, "An Application-Specific Protocol Architecture for Wireless Microsensor Networks", IEEE Trans. Wireless Communication, vol. 1, no. 4, October 2002, pp. 660-70. https://doi.org/10.1109/TWC.2002.804190

[8] Ravneet Kaur, Deepika Sharma and Navdeep Kaur. "Comparative Analysis of Leach and Its Descendant Protocols in Wireless Sensor Network". International Journal of P2P Network Trends and Technology- Volume3, Issue1- 2013.

[9] G. Smaragdakis, I. Matta, A. Bestavros. "A Stable Election Protocol for Clustered Heterogeneous Wireless Sensor Networks", Proceedings of the 2nd International Workshop on SANPA 2004, Massachusetts, U.S, 2004, pp: 1-11.

[10] Heena Dhawan, Sandeep Waraich "A Comparative Study on LEACH Routing Protocol and its Variants in Wireless Sensor Networks: A Survey", International Journal of Computer Applications (0975 - 8887) Volume 95- No.8, June 2014, pp: 21-27.https: //doi.org/10.5120/16614-6454

[11] NEETIKA, SIMARPREET KAUR, "Review on Hierarchical Routing in Wireless Sensor Networks", in International Journal of Smart Sensors and Ad- Hoc Networks (IJSSAN), Vol. 2, Issue 3, 4, 2012, pp. 85-90.

[12] Thomas Preud Homme, Julien Sopena, Gaël Thomas, Bertil Folliot. Batch Queue: « file producteur / consommateur optimisée pour les multi-cœurs ». 8ème Conférence Française sur les Systèmes d'Exploitation (CFSE'11), May 2011, Saint-Malo, France. Pp.1-12, 2011.

\section{$7 \quad$ Authors}

Yassine Rayri is Ph.D. candidate in Laboratory Information Modeling and Communication Systems (MISC), Ibn Tofail University, Morocco. He received the specialized Master's degree in systems and networks from the Faculty of Sciences.

Hatim Kharraz Aroussi is a research professor in MISC, her research activities have been concerned with Sensor Networks, Sensor Location, Energy Consumption Sensors, and Sensor Network Security.

Abdelaziz Mouloudi is a research professor in MISC, her research activities have been concerned with Computer Security, Cryptography, and Evolutionary Algorithms.

Article submitted 07 May 2018. Resubmitted 22 May 2018. Final acceptance 30 June 2018. Final version published as submitted by the authors. 